\title{
Analysis of VANET Wireless Networking Technologies in Realistic Environments
}

\author{
Muhammad Naeem Tahir \\ Finnish Meteorological Intitute, Arctic \\ Space Center (FMI-ARC) \\ Erik Palmenin aukio 1, Helsinki, Finland \\ naeem.tahir@fmi.fi
}

\author{
Marcos Katz \\ Centre for Wireless Communications \\ (CWC) - Networks \& Systems \\ University of Oulu, Finland \\ marcos.katz@oulu.fi
}

\author{
Urooj Rashid \\ Helsinki Institute of Sustainability Science \\ (HELSUS) \\ University of Helsinki, Fin land \\ urooj.rashid@helsinki.fi
}

\begin{abstract}
Communication is essential to provide an intelligent services to connected cars. For operational services, connected vehicles in VANET (Vehicle Ad hoc Networks) regularly try to transfer large amounts of data for vehicular safety. Similarly, V2x (Vehicle-to-Everything) communication includes vehicles transferring information with each other and with infrastructure i.e., Vehicle-to-Infrastructure (V2I) and Vehicle-to-Vehicle (V2V) are proven to increase traffic safety and security as well as to enhance the efficiency of intelligent transportation system (ITS). Vehicular connectivity is provided using short-range technologies such as the IEEE 802.110 standard or cellular approaches, such as the $5 \mathrm{G}$ network. In this article, we consider combining these technologies in a cooperative manner and aiming at exploiting jointly their advantages. In this cooperative heterogeneous network, the IEEE 802.11p supports safety-related pilot use cases while the provision of non-safetyrelated pilot use cases are supported by the 5G test network.
\end{abstract}

Keywords-ITS, IEEE-802.11p, 5G, V2V, V2I

\section{INTRODUCTION}

In the last decade, the vehicular communications have become more useful and appealing as they promise to significantly decrease road mortalities, allowing safe mobility and supporting intelligent vehicular systems. The ITS implies solutions devised to improve mobility, reduce traffic jamming with increased efficiency and enhance safety for road transport, offering safe driving with increased environmental friendliness. In an ITS system, the safety critical applications are truly essential in vehicular communications. Lately, ITS applications were mounted in both on the road infrastructure i.e., allowing safe traffic management and in vehicles for numerous purposes such as driver assistance. Both methodologies offer considerable benefits for ITS system.

Since many years, the wireless technology of choice for V2V and V2I communications has been IEEE-802.11p. Lately, a new wireless standard started to evolve under the umbrella of the 3rd generation partnership project (3GPP), addressing V2x safety applications that majorly emphasized on mobile broadband standardization [1]. The evolution of IEEE $802.11 \mathrm{p}$ and 3GPP technology standardization is presented in Fig.1. Since the safety of billions of people on roads will depend on the practical performance of these VANET wireless technologies, it is highly relevant to compare them and make an analysis of these technologies in different vehicular environments [2]. In this paper, we study the coexistence of short-range IEEE $802.11 \mathrm{p}$ and cellular based $5 \mathrm{G}$ network. Exploiting these technologies in a cooperative manner results in a heterogeneous system for vehicular networking that leverages the best of both technologies. In this cooperative heterogeneous network, the IEEE $802.11 \mathrm{p}$ supports safetyrelated pilot use cases while the provision of non-safety-related pilot use cases are supported by the $5 \mathrm{G}$ test network.

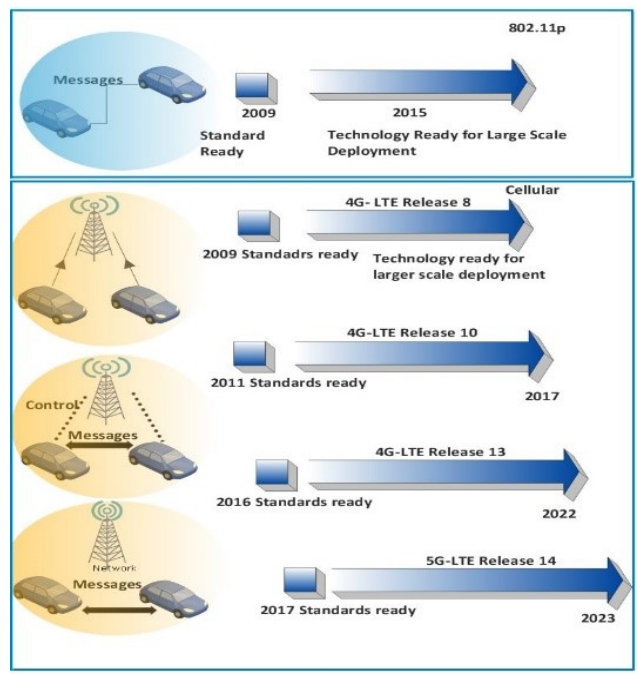

Fig. 1 Evolution of vehicular networking technologies.

\section{VANET WIRELESS TECHNOLOGIES}

\section{A. IEEE $802.11 p$}

The modified version of the IEEE standard 802.11 for WiFi communications were established during 2005 to 2010 to meet the lacking requirements in the communication system. The IEEE 802.11 improves the physical communication layer in terms of developed WAVE (Wireless Access in Vehicular Environments) and ETSI (European Telecommunications Standards Institute) ITS-G5 standards [3]. The respective technologies have proven to meet all the requirements in vehicular communication system as the extensive field operational trials have been performed in various countries of Europe and US as well. Meanwhile the industry has developed transceiver chipsets allowing mass production and further deployment at large scale. The communication with the bandwidth of $5.9 \mathrm{GHz}$ band has been allocated in both US and Europe. More demanding requirements on environmental and traffic conditions were later required. Because of this, governmental institutions started to use roadside infrastructures including sensors and cameras to collect the expected data about the environmental and traffic conditions [4]. The 
802.11p provides the best available features for the vehicular communication approach. It was intended to meet various $\mathrm{V} 2 \mathrm{x}$ application requirements with the most advanced specifications. The IEEE $802.11 p$ can clearly decrease the number of collisions on the road. However, IEEE $802.11 \mathrm{p}$ significantly increases the communication load on network. This is primarily because of the high message frequency requirements of safety applications in a scarce and shared wireless spectrum. The IEEE $802.11 \mathrm{p}$ deals with the congestion control techniques such as contention window adaptations, message frequency and transmit power to all the medium access control (MAC) layers. It allows an uncoordinated and fully distributed access to the wireless channel with no compulsion of resource allocation procedure [5]. The Physical Layer (PHY) is used with eight possible combinations of Modulation and Coding Schemes (MCSs) called modes. Focusing on the adopted MCS, the rate of data varies between 3-54 Mbps.

\section{B. $5 G$ Wireless network}

$5 \mathrm{G}$ wireless networks and the ITS infrastructure can lead to the establishment of intelligent cities and roads with the deployment of ultra-low latency networks for the sharing of real-time data between both vehicles and transport infrastructure [6]. The VANET's faced the challenge for the high-mobility of autonomous vehicles to detect other vehicles. Moreover, the vehicles in VANET's also needs the enough time to respond to the possible threats that may be encountered. The deployment of $5 \mathrm{G}$ wireless communication systems allows communication to alert traffic management systems to enable and modify the patterns of the traffic and provide the optimal routes to vehicles and efficiently guiding emergency services to their destination. 5G network infrastructures promise to provide a much-advanced platform by upgrading the cellular technologies by combining the connectivity, shared standards, wireless communication and Internet of things (IoT). Future technologies will provide smoother upgrade paths that will aid to provide up-to-date and stronger services including security to protect both the intelligent transportation systems and the wireless networks themselves. These advanced technologies will get more sophisticated in future, so will the equipment that allows hackers to exploit their vulnerabilities and making software and security updates possibly easier process in everyone's interest [7].

\section{TEST LOCATIONS}

Two test locations (Petäjämaa \& Sod5g test track) were used at the Finnish Meteorological Institute (FMI) to develop road weather and vehicular network piloting, as presented in Fig. 2.

The Petäjämaa test location was used for the following tasks.

1. Typical road weather station measurements

2. Special measurements for research purposes

3. Supported VANET (vehicular ad hoc network) protocols
4. Measurements of road temperature, air temperature, wind speed and direction, weather cameras.

5. Measurement of visibility, frost depth, friction (both RWS and vehicle)

\section{IEEE 802.11p, IEEE 802.11n/g Wi-Fi, 4G/5G}

The supported devices used for the measurements include vehicular PCs (Sunit), conventional laptops, Android tablets and phones.

The Sod5G test track http://sod5g.fmi.fi is composed of a 5G-test network working at $2.3 \mathrm{GHz}$ band [8]. It is based on LTE-A hardware and connected to 5GTN. The Sod5G test track also supports vehicle test network IEEE 802.11p and it also has a road weather infrastructure (2 RWS). The Sod5G versatile vehicle winter testing track has a $1.7 \mathrm{~km}$ main track (gravel/concrete) as presented in the Fig. 2.

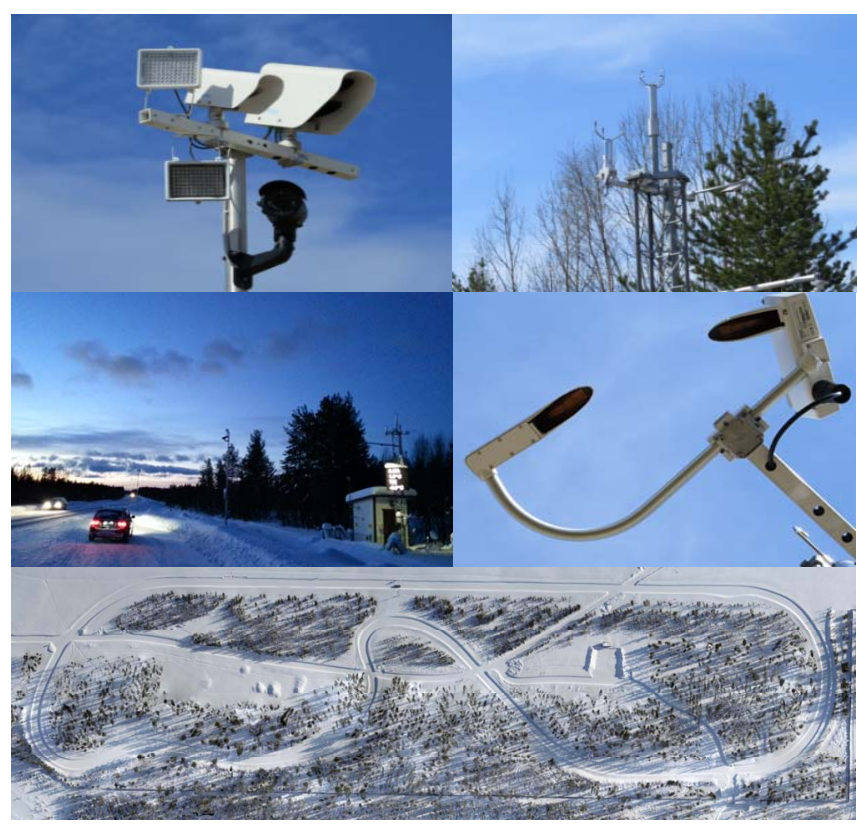

Fig. 2. Test track eqipeed with testing infrstructure

\section{COMPARISON OF CELLULAR VS DSRC}

In this comparison, the $5 \mathrm{G}$ test network is slightly more sensitive to the situation where a user receives signals from more than one transmitter with different level of power i.e., the near-far issue, as illustrated in Fig 3. The dissimilarity in power level may occur even for two near-by wireless transmitters, although one of the transmitters (Tx) is obstructed. Meanwhile, IEEE $802.11 \mathrm{p}$ permits each user to transmit in a distinct OFDM symbol, and situationally, the receiver adjusts its parameters accordingly i.e., automatic gain controller (AGC), time off-set estimation and frequency offset estimation in an optimum way for each user autonomously. On the other hand, the $5 \mathrm{G}$ test network offers the same resources for users within similar OFDM symbol but the receiver (Rx) adjusts its AGC gain depending on a particular combined signal. Hence, in the 
$5 \mathrm{G}$ test network, the capacity of the receiver $(\mathrm{Rx})$ recognizing weak messages in the presence of strong messages is restricted [8].

Here, the weak messages may have higher importance than the strong one. For example, considering the TCP, an important message with low power could be received from a vehicle transmitter with delay, and an earlier received message with good power is neglected. The low relevance to good power messages with safety decisions might impose a real risk.

Table I shows a comparison between different vehicular wireless protocols The $5 \mathrm{G}$ test network has some advantages over IEEE-802.11p in terms of range, as shown in Table I. IEEE $802.11 \mathrm{p}$ requires the deployment of several access points (APs) and gateways that escalates the cost and time of deployment. One of the challenges that operators face is to cover the deployment costs of APs with a not at all clear sight of business model [9].

TABLE I. WIRELESS TRANSMISSION PROTOCOLS COMPARSION

\begin{tabular}{|l|l|l|}
\hline Description & $\begin{array}{l}\text { DSRC (IEEE- } \\
\mathbf{8 0 2 . 1 1 p}\end{array}$ & 5G Test Network \\
\hline Range $(\mathrm{m})$ & 900 & 1500 \\
\hline Jitter $(\mathrm{ms})$ & 3.76 & 5.30 \\
\hline $\begin{array}{l}\text { Network Latency } \\
(\mathrm{ms})\end{array}$ & 48.39 & 70.79 \\
\hline Cyclic Prefix (CP) & $1.72 \mu \mathrm{s}$ & $4.58 \mu \mathrm{s}$ \\
\hline $\begin{array}{l}\text { Reason of Cyclic } \\
\text { Prefix }(\mathrm{CP})\end{array}$ & Delay Spread & $\begin{array}{l}\text { Propagation Interval \& Delay } \\
\text { Spread, Timing errors }\end{array}$ \\
\hline
\end{tabular}

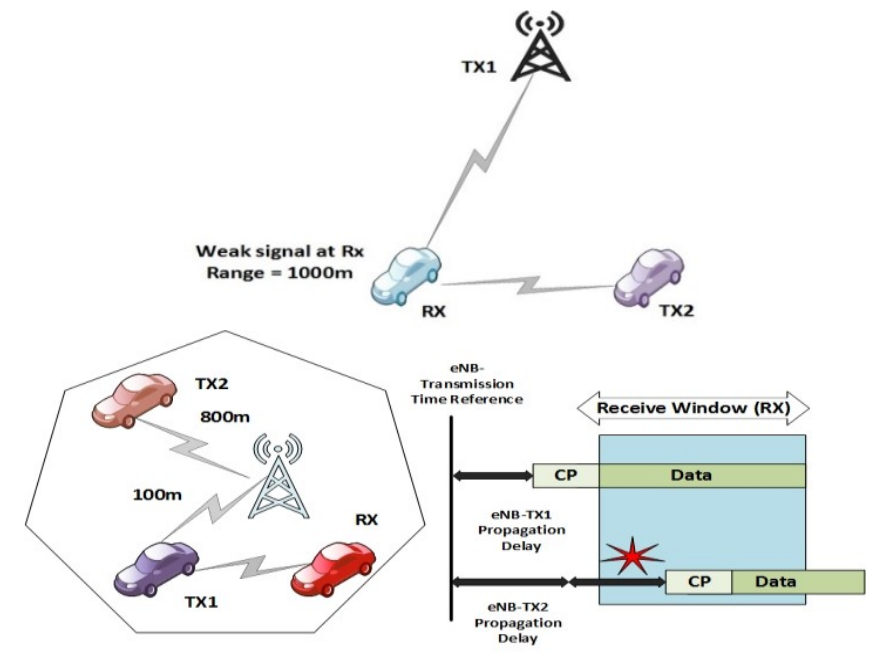

Fig. 3. ITS protocols effect of cyclic prefix in distance

One way to compare V2x wireless technologies i.e., IEEE$802.11 \mathrm{p}$ and $5 \mathrm{G}$ can be done by considering real-time performance tests in outdoor conditions under similar environments. The above-mentioned comparative Table I shows a 1500 range for the $5 \mathrm{G}$ test network. Unfortunately, $5 \mathrm{G}-\mathrm{V} 2 \mathrm{x}$ complete pilot use-cases are not presented yet for comparison. Meanwhile, the synchronization idea for IEEE$802.11 \mathrm{p}$ puts a restriction on the communication range in vehicular communication that is revealed in the changed role allocated to the cyclic prefix (CP), as presented in Fig. 3 and Table I. The network latency and jitter measurements reveal that $5 \mathrm{G}$ still needs time to roll-out completely for $\mathrm{V} 2 \mathrm{x}$ applications, as compared to IEEE-802.11p.

\section{CONCLUSION}

In VANET's the communication between vehicles and between susceptible road users are anticipated to offer considerable assistances in terms of vehicular safety. It also assists to develop and improve road traffic management. The main objective of this article is to make a comparative analysis between IEEE $802.11 \mathrm{p}$ and the next generation 5G test network in VANET's. The comparative study of vehicular networking standards includes latency, coverage, and cyclic prefix. Our result indicates that IEEE $802.11 \mathrm{p}$ offers reasonable performance with restricted mobility and support. However, 5G also meets many of the requirements for vehicular applications regarding latency, coverage, and cyclic prefix. Hence, the intended expansion of V2x facilities in $5 \mathrm{G}$ makes much sense and also holds potential for future applications. Meanwhile, numerous ITS applications based on IEEE-802.11p further develop and can take off.

\section{ACKNOWLEDGEMENT}

Here I would like to say thanks to Timo Sukuvaara and Kari Mäenpää from Finnish Meteorological Institute (FMI), Finland, for their support and guidance in the abovementioned measurements.

\section{REFERENCES}

[1] Ojanperä, Tiia, et al. "Development and Piloting of Novel 5G-Enabled Road Safety Services." 2019 IEEE Wireless Communications and Networking Conference Workshop (WCNCW). IEEE, 2019.

[2] Tahir, M., Mäenpää, K., \& Sukuvaara, T. (2020). Performace Evaluation of Vehicular Communication, Transport and Telecommunication Journal, 21(3), 171-180. doi: https://doi.org/10.2478/ttj-2020-0013

[3] Ucar, Seyhan, Sinem Coleri Ergen, and Oznur Ozkasap. "Multihopcluster-based IEEE 802.11 p and LTE hybrid architecture for VANET safety message dissemination." IEEE Transactions on Vehicular Technology 65.4 (2015): 2621-2636

[4] Festag, A. (2015). Standards for vehicular communication-from IEEE $802.11 \mathrm{p}$ to 5G. e \& i Elektrotechnik und Informationstechnik, 132(7), 409-416.

[5] Cecchini, Giammarco et al. "Performance comparison between IEEE $802.11 \mathrm{p}$ and LTE-V2V in-coverage and out-of-coverage for cooperative awareness.” 2017 IEEE Vehicular Networking Conference (VNC) (2017): 109-114.

[6] Zhou, Haibo, et al. "Evolutionary V2X technologies toward the Internet of vehicles: Challenges and opportunities." Proceedings of the IEEE 108.2 (2020): 308-323.

[7] Yuan, Weijie, et al. "Distributed Estimation Framework for Beyond 5G Intelligent Vehicular Networks." IEEE Open Journal of Vehicular Technology 1 (2020): 190-214.

[8] Timo Sukuvaara, "ITS-Enabled advanced road weather services and infrastructures for vehicle winter testing, professional traffic fleets and future automated driving", in proceedings of ITS World Congress in Copenhagen, September 17.-21. 2018 Copenhagen, Denmark

[9] Arena, Fabio, Giovanni Pau, and Alessandro Severino. "V2X Communications Applied to Safety of Pedestrians \& Vehicles." Journal of Sensor and Actuator Networks 9.1 (2020): 3. 